\title{
Study on Rayleigh Wave Inversion for Estimating Shear-wave Velocity Profile
}

\author{
T.A. Sanny*, Yoes Avianto* \\ *Geophysical Engineering Department, Faculty of Earth Science and Mineral \\ Technology, Institut Teknologi Bandung \\ Jalan Ganesha 10 Bandung 40135, Phone/fax: (62) (22) 2509168 \\ E-mail: sanny@gf.itb.ac.id
}

\begin{abstract}
Rayleigh wave or ground roll is a noise in seismic body waves. However, how to use this noise for soil characterization is very interesting since Rayleigh wave phase velocity is a function of compression-wave velocity, shearwave velocity, density and layer thickness. In layered-medium Rayleigh wave velocity also depends on wavelength or frequency, and this phenomenon is called dispersion. Inversion procedure to get shear-wave velocity profile needs a priori information about the solution of the problem to limit the unknown parameters. The Lagrange multiplier method was used to solve the constrained optimization problems or well known as a smoothing parameter in inversion problems. The advantage of our inversion procedure is that it can guarantee the convergence of solution even though the field data is incomplete, insufficient, and inconsistent. The addition of smoothing parameter can reduce the time to converge. Beside numerical stability, the statistical stability is also involved in inversion procedure. In field experiment we extracted ground roll data from seismic refraction record. The dispersion curves had been constructed by applying $\mathrm{f}-\mathrm{k}$ analysis and $\mathrm{f}-\mathrm{k}$ dip filtering. The dispersion curves show the dependence of Rayleigh wave phase velocities in layered media to frequency. The synthetic models also demonstrate the stability and the speed of inversion procedure.
\end{abstract}

\section{$1 \quad$ Introduction}

Seismic methods in geophysical exploration basically take a wave velocity as unknown parameter. Once we have the information of wave velocity through a material, we should determine elastic properties of materials for purpose of geotechnical or environmental studies. The seismic methods have been developed for many years, such as seismic refraction methods, reflection methods, seismic borehole methods, and seismic tomography methods (Kaneko et al., 1990). There are two main types of wave velocity which are discovered in seismic methods, compressional-wave and shear-wave velocities. Many efforts are spent to relate shear-wave and compressional wave velocities with the elastic properties of material. 
Another method which comes up in nowadays is seismic surface-waves, including Rayleigh-wave (e.g Mokhtar et al., 1988). Rayleigh wave is a kind of surface waves which travels along free-surface and is a result of interference of compressional and shear waves. Particle of this wave moves in a retrograde elliptical way and its amplitude decreases according to depth (Sheriff and Geldart, 1995; Rikitake et al., 1987). Basically we can extract Rayleigh waves into two kinds of wave which are compressional and shear-vertical waves. This fact gives the idea of using Rayleigh wave in geophysical exploration to estimate both compressional and shear wave velocities. However how to pick Rayleigh wave data from deeper layer is more difficult than body waves.

Jones (1962) firstly implemented this method for measuring the thickness and elastic properties of roads. He conducted the forward modelling and simplified the equation for lowest half-space liquid. Nazarian and Stokoe (1983) presented the acquisition data of Rayleigh wave, called Spectral Analysis of Surface Waves (SASW), using two geophones along a straight line. Following the SASW method, multichannel record had also been proposed by Park et al (1999) and Foti (2000). In this paper, we will give a brief overview explanation about how to use Rayleigh wave in geophysical exploration.

\section{Characteristic Equation of Rayleigh-waves}

In a semi-infinite medium, Rayleigh wave is not dispersive. It means that it does not depend on frequency or wavelength. Sheriff and Geldart (1995) showed that the velocity of Rayleigh waves is approximately $0.919 \beta$ (where $\beta$ is shear wave velocity) for a Poisson's ratio of 0.25 .

The mathematical formula of Rayleigh waves in layered media is more complicated. Ewing et al (1957) provided the derivation of Rayleigh wave propagation and presented a matrix $4 \mathrm{n}-2$ order for $\mathrm{n}$-th layered system. This matrix method is not recommended in computation because of its large order. Thomson (1950, op cit. Aki and Richards, 1980) and Haskell (1953, op cit. Aki and Richards, 1980) presented more simplified matrix to solve this problem, called propagator matrix method. They made an assumption that the vertical heterogeneous media are made of a stack of homogeneous layers lying above semi-infinite medium. This kind of matrix is to solve the eigenvalueeigenvector problem of first differential wave equation as follows (Aki and Richards, 1980):

$$
\frac{\mathrm{d}}{\mathrm{dz}}\left[\begin{array}{c}
\mathrm{r}_{1} \\
\mathrm{r}_{2} \\
\mathrm{r}_{3} \\
\mathrm{r}_{4}
\end{array}\right]=\left[\begin{array}{cccc}
0 & \mathrm{k} & \mu^{-1}(\mathrm{z}) & 0 \\
-\mathrm{k} \lambda(\mathrm{z})[\lambda(\mathrm{z})+2 \mu(\mathrm{z})]^{-1} & 0 & 0 & {[\lambda(\mathrm{z})+2 \mu(\mathrm{z})]} \\
\mathrm{k}^{2} \zeta(\mathrm{z})-\omega^{2} \rho(\mathrm{z}) & 0 & 0 & \mathrm{k} \lambda(\mathrm{z})[\lambda(\mathrm{z})+2 \mu(\mathrm{z})]^{-1} \\
0 & -\omega^{2} \rho(\mathrm{z}) & -\mathrm{k} & 0
\end{array}\right] \cdot\left[\begin{array}{l}
\mathrm{r}_{1} \\
\mathrm{r}_{2} \\
\mathrm{r}_{3} \\
\mathrm{r}_{4}
\end{array}\right]
$$


where $\zeta(\mathrm{z})=4 \mu(\mathrm{z})[\lambda(\mathrm{z})+\mu(\mathrm{z})] /[\lambda(\mathrm{z})+2 \mu(\mathrm{z})], \mu(\mathrm{z})$ and $\lambda(\mathrm{z})$ are Lame's constants as a function of depth, $\rho(z)$ is density as a function of depth, $k$ is wavenumber, and $\omega$ is angular frequency. $\left(r_{1}, r_{2}, r_{3}, r_{4}\right)$ is motion-stress vector. Equation (1) has the solution:

$$
\left[\begin{array}{l}
\mathrm{r}_{1} \\
\mathrm{r}_{2} \\
\mathrm{r}_{3} \\
\mathrm{r}_{4}
\end{array}\right]=\mathbf{F w}=\mathbf{F}\left[\begin{array}{c}
\mathrm{P} \\
\mathrm{S} \\
P \\
S
\end{array}\right]
$$

where $(\mathrm{P}, \mathrm{S}, P, S)$ is displacement amplitude of $\mathrm{P}$ - and $\mathrm{S}$-wave and $\mathbf{F}$ is a matrix which is made up from eigenvectors of equation (1) multiplied by the diagonal matrix containing the vertical phase factors (Aki and Richards, 1980).

Using the propagator matrix to solve the equation (2), we can relate the amplitude of different wave-types in the lowermost half-space to the motionstress vector at the free-surface:

$$
\mathbf{w}_{\mathrm{n}+1}=\mathbf{F}_{\mathrm{n}+1}{ }^{-1} \mathbf{P}\left(\mathrm{z}, \mathrm{z}_{0}\right) \mathbf{r}\left(\mathrm{z}_{0}\right)=\operatorname{Br}\left(\mathrm{z}_{0}\right)
$$

where $\mathbf{P}\left(z, z_{0}\right)$ is the propagator matrix and $\mathbf{B}$ is matrix $4^{\text {th }}$ order consisting of propagator matrix time $\mathbf{F}$. Thus the eigenvalue of matrix $\mathbf{B}$ is given by (Aki and Richards, 1980):

$$
\left|\begin{array}{ll}
\mathrm{B}_{31} & \mathrm{~B}_{32} \\
\mathrm{~B}_{41} & \mathrm{~B}_{42}
\end{array}\right|=0
$$

Equation (4) is known as the characteristic equation of Rayleigh wave or dispersion equation of Rayleigh wave which can be written as a non-linear, implicit form:

$$
f\left[\alpha(\mathrm{z}), \beta(\mathrm{z}), \rho(\mathrm{z}), \mathrm{f}_{\mathrm{j}}, \mathrm{c}_{\mathrm{j}}\right]=0
$$

where $\alpha(z)$ is compressional wave velocity, $\beta(z)$ is shear wave velocity, $\rho(z)$ is density and all parameters above are a function of depth. $f_{j}$ is frequency, and $c_{j}$ is Rayleigh wave phase velocity at frequency $f_{j}(j=1,2,3 \ldots, n)$. We will solve this equation only for single layer and two layers system lying on semi-infinite medium.

\section{Forward Modelling : Dispersion Curves Construction}

Since the dispersion equation (equation 5) is a function of frequency, we can construct the dispersion curves of Rayleigh wave. Given the parameter $\alpha, \beta, \rho$, 
and $\mathrm{H}$, the Rayleigh wave phase velocity can be constructed in a frequency range. We present here only in a fundamental mode. To find the root of dispersion equation, we use a simple bisection method (Chapra and Canale, 1998) and define the range where the roots exist.

Given parameters for first layer $\beta_{1}=300 \mathrm{~m} / \mathrm{s}, \alpha_{1}=560 \mathrm{~m} / \mathrm{s}, \rho_{1}=1 \mathrm{~g} / \mathrm{cm}^{3}$, and for semi-infinite layer $\beta_{2}=500 \mathrm{~m} / \mathrm{s}, \alpha_{2}=935 \mathrm{~m} / \mathrm{s}, \rho_{2}=1 \mathrm{~g} / \mathrm{cm}^{3}$, and layer thickness $\mathrm{H}=5 \mathrm{~m}$, the dispersion curves can be seen in Figure 1 .

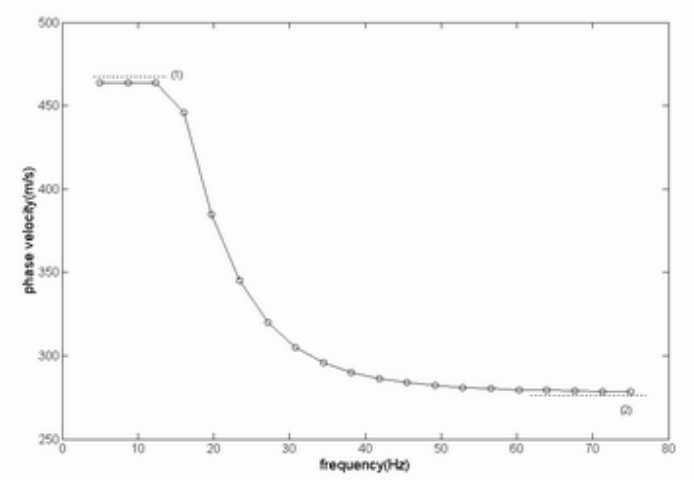

Figure 1 Dispersion curves for two-layer model.

It is seen that the dispersion curve has two asymtots in low and high frequencies which define that the range of Rayleigh wave phase velocity will not exhibit the shear wave velocity in each layer. Similar dispersion curve has been found in a

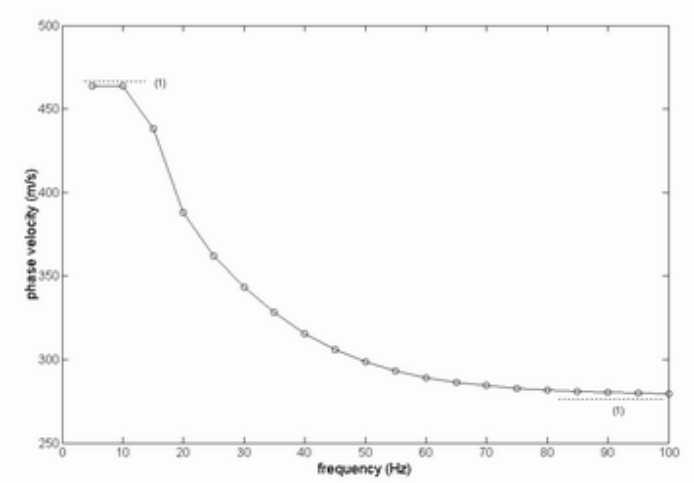

Figure 2 Dispersion curve for a three-layer model.

three-layer model (Figure 2). Consider three-layer model with the parameters of $1^{\text {st }}$ layer are $\beta_{1}=300 \mathrm{~m} / \mathrm{s}, \alpha_{1}=560 \mathrm{~m} / \mathrm{s}, \rho_{1}=1.8 \mathrm{~g} / \mathrm{cm}^{3}$, the parameters of $2^{\text {nd }}$ layer are $\beta_{2}=400 \mathrm{~m} / \mathrm{s}, \alpha_{2}=750 \mathrm{~m} / \mathrm{s}, \rho_{2}=1.8 \mathrm{~g} / \mathrm{cm}^{3}$, the parameter of semi- 
infinite layer are $\beta_{3}=500 \mathrm{~m} / \mathrm{s}, \alpha_{3}=935 \mathrm{~m} / \mathrm{s}, \rho_{3}=1.8 \mathrm{~g} / \mathrm{cm}^{3}$, and layer thickness $\mathrm{H}_{1}=3 \mathrm{~m}$ and $\mathrm{H}_{2}=3 \mathrm{~m}$, the dispersion curve is shown in Figure 2 .

\section{$4 \quad$ Inversion Procedure}

The inversion produre basically estimates the shear wave velocity from dispersion curve (furthermore see Xia et al., 1999). It is often ill-posed or unstable, especially non-linear problems such as Rayleigh wave inversion. To solve this problem, adding a priori information about the solution of the problem is important to limit the unknown parameters. A smoothing parameter is also used to guarantee the inversion procedure to converge. This method is related to the Lagrange multiplier method to solve the constrained optimization problems.

To incorporate the priori information to the problems, the necessary constraining equation may be developed in the form $\mathbf{D m}=\mathbf{h}$, where $\mathbf{D}_{\mathrm{pxp}}$ is an operator matrix that operates on the $\mathrm{p}$ parameter, $\mathrm{m}$ to yield or preserve the priori information data contained in vector $\mathbf{h}$. If there is no informative a priori information, vector $\mathbf{h}$ is set equal to zero. Mathematically, we wish to minimize the Lagrangian function: (Meju, 1994)

$$
L=(\mathbf{W c}-\mathbf{W f}(\boldsymbol{\beta}))^{T}\left(\mathbf{W c}-\mathbf{W} \mathbf{f}(\boldsymbol{\beta})+\mu \boldsymbol{\beta}^{T} \mathbf{D}^{T} \mathbf{D} \boldsymbol{\beta}\right.
$$

Because the problem is incomplete, insufficient, and inconsistent field data, we find the smoothest as judged by the measure $\boldsymbol{\beta}^{\mathrm{T}} \mathbf{D}^{\mathrm{T}} \mathbf{D} \boldsymbol{\beta}$ amongst all possible solutions with residual $|\mathbf{c}-\mathbf{f}(\beta)|^{2}$ (Meju, 1994). So we can guarantee the convergence of solution. Then, the solution for the parameter correction is:

$$
\Delta \beta=\left(\left(\mathbf{W} . \mathbf{J}_{\beta}\right)^{T}\left(\mathbf{W} . \mathbf{J}_{\beta}\right)+\mu^{2} \mathbf{D}^{T} \mathbf{D}\right)^{-1}\left(\left(\mathbf{W} . \mathbf{J}_{\beta}\right)^{T}(\mathbf{W y})-\mu^{2} \mathbf{D}^{T} \mathbf{D} \boldsymbol{\beta}^{0}\right.
$$

where $\mathbf{J}_{\beta}$ is the Jacobian matrix which respect to shear-wave velocity parameter $(\beta), \boldsymbol{\beta}$ is the vector of shear wave velocity, $\mathbf{y}$ is the difference between observational data and model data, $\mu$ is smoothing parameter, $\mathbf{W}$ is weighting matrix which consists of standard deviation of observational data. $\mathbf{D}$ is a smoothness matrix defined by (Meju, 1994):

$$
\mathrm{D}=\left[\begin{array}{cccccc}
1 & -1 & & & & \\
& 1 & -1 & & & \\
& & & \cdot & & \\
& & & & \cdot & \\
& & & & 1 & -1
\end{array}\right]
$$


The stability of the inversion procedure is achieved by incorporating constrains on parameters and smoothness factor.

The standard deviation of data mode can be derived from square root of diagonal elements of parameter covariance matrix of the last iteration with the following relationship (Meju, 1994):

$\operatorname{Cov}(\beta)=\left(\mathbf{J}_{\beta}{ }^{T} \mathbf{W}^{T} \mathbf{W} \mathbf{J}_{\beta}+\mu^{2} \mathbf{D}^{T} \mathbf{D}\right)^{-1} \mathbf{J}_{\beta}{ }^{T} \mathbf{W}^{T} \mathbf{W} \mathbf{J}_{\beta}\left(\mathbf{J}_{\beta}{ }^{T} \mathbf{W}^{T} \mathbf{W} \mathbf{J}_{\beta}+\mu^{2} \mathbf{D}^{T} \mathbf{D}\right)^{-1}$ (8)
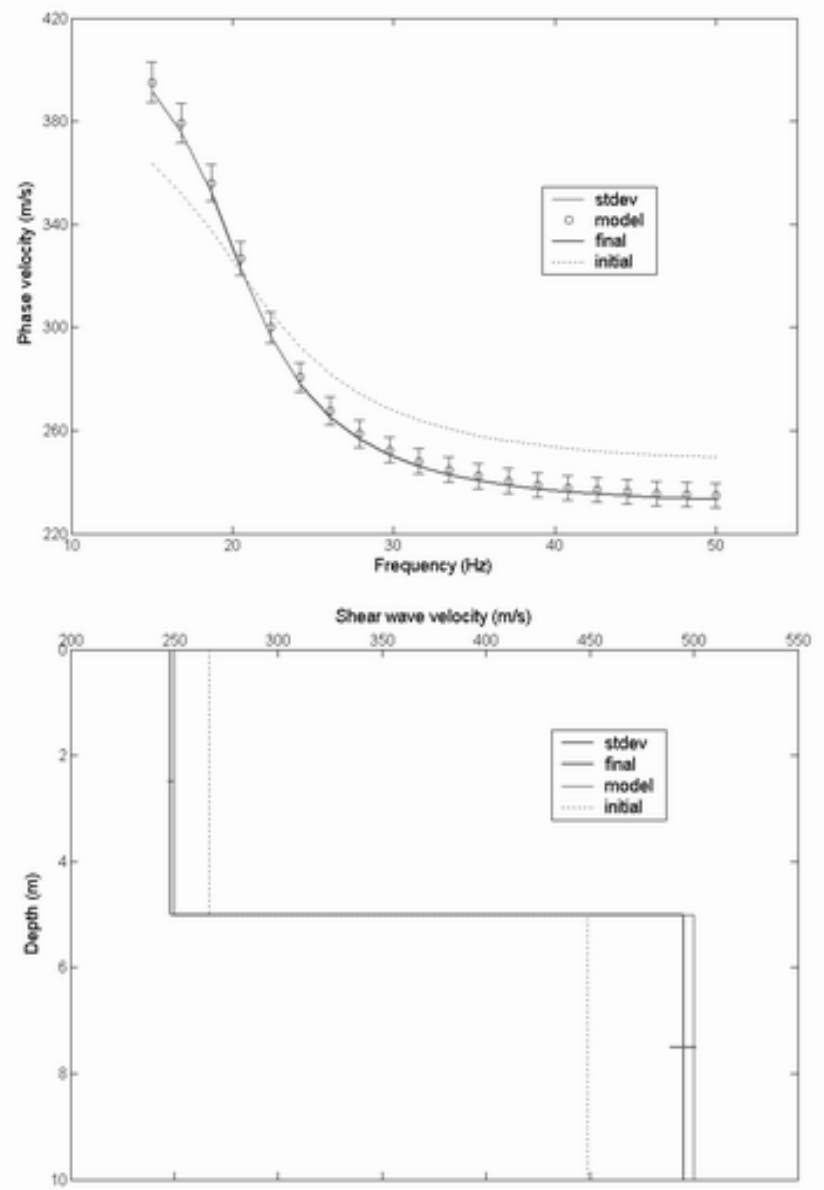

Figure 4 Inversion result for two-layer model. 


\section{$5 \quad$ Synthetic Models}

The inversion algorithm above has been applied using synthetic models and only shear-wave velocity was involved in jacobian matrix. For twolayer model with first layer thickness $5 \mathrm{~m}$, we set the shear wave velocity for the case with weathered zone whose the shear wave velocities are 250 $\mathrm{m} / \mathrm{s}$ and $500 \mathrm{~m} / \mathrm{s}$. With correct initial values, we have one iteration to reduce the rms error from 3.0 to 0.4 (Figure 4 ).

For three-layer model with $\beta_{1}=300 \mathrm{~m} / \mathrm{s}, \beta_{2}=400 \mathrm{~m} / \mathrm{s}, \beta_{3}=500 \mathrm{~m} / \mathrm{s}$, and layer thickness $\mathrm{H}_{1}=3 \mathrm{~m}, \mathrm{H}_{2}=3 \mathrm{~m}$, the rms error reduced from 6.3 to 0.02 after four iterations (Figure 5). The initial value was set to be $25 \%$ of the model value.
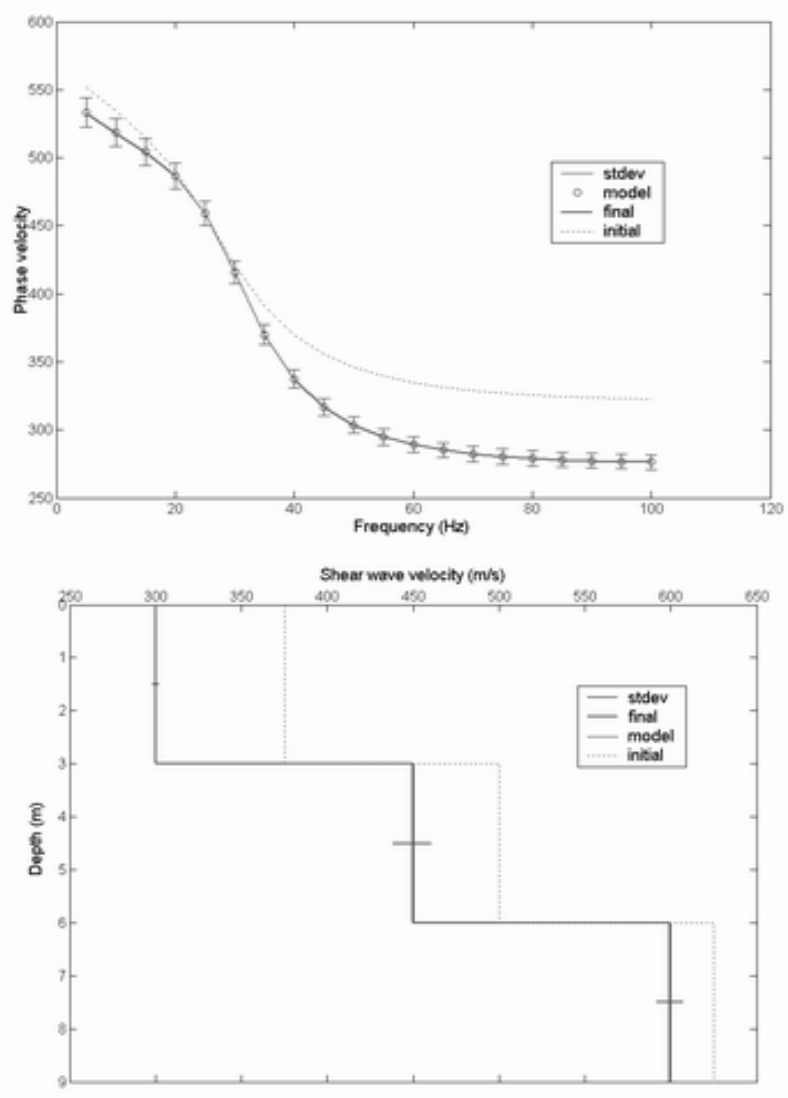

Figure 5 Inversion result for a three-layer model. 


\section{$6 \quad$ Field Experiment}

The synthetic model has been implemented in the field experiment. Surfacewave data were acquired using the conventional refraction seismic method in Dago Pakar Resort, northern Bandung, West Java, Indonesia on March, 2002. There were two layers of soil, weathering zone and volcanic breccia, which can be seen on the side of the hill-slope.

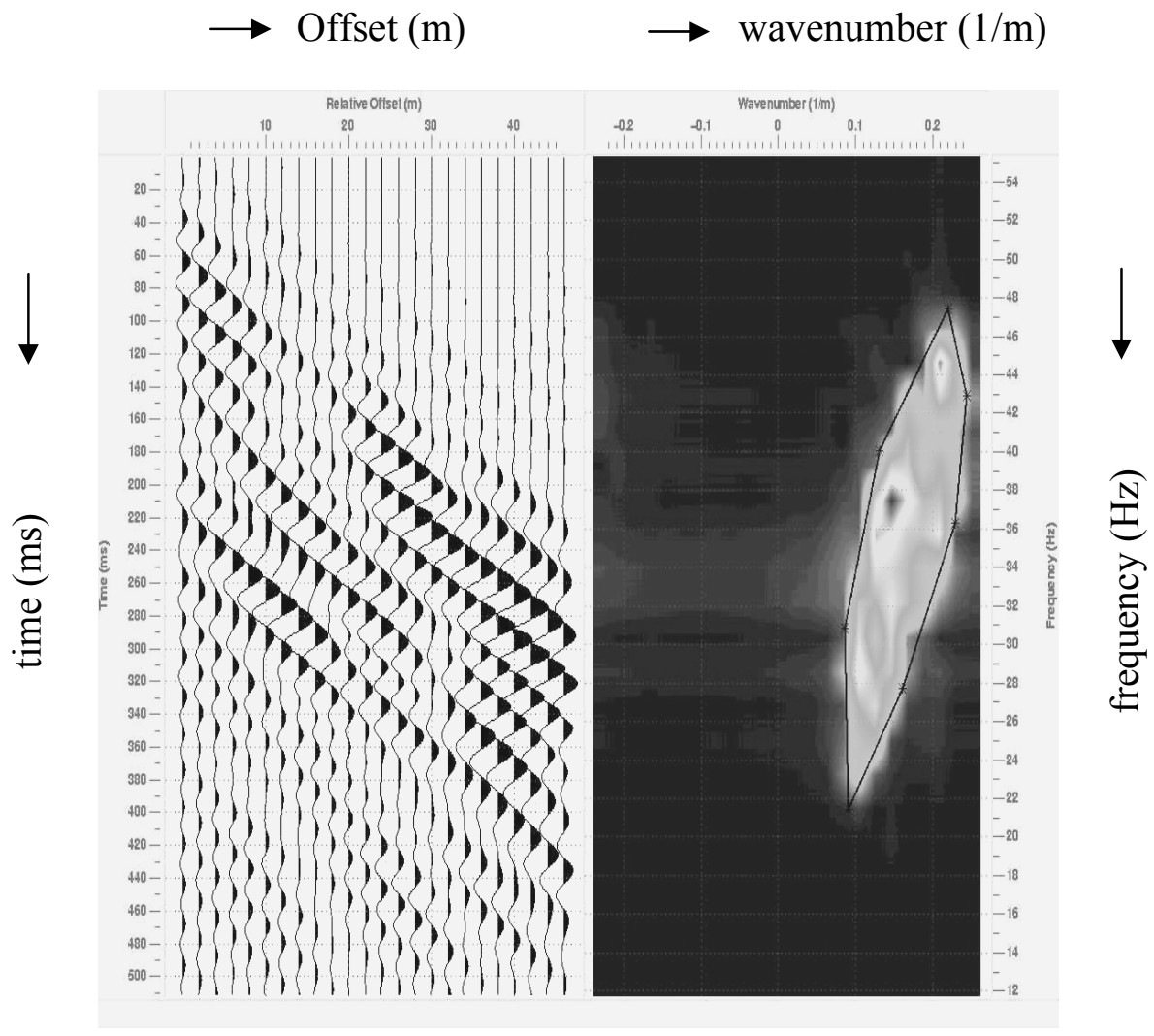

Figure 6 Ground roll after dip filtering (near offset $10 \mathrm{~m}$ ).

Hammer was used as an energy source in refraction seismic method. 24 vertical geophones available on Geophysical Engineering Department, ITB, were arranged with $2 \mathrm{~m}$ intervals, with near-offset $10 \mathrm{~m}$. For comparing the result from refraction method, we also conducted a downhole method. 9 vertical geophones were arranged on the side of the slope hill with $0.5 \mathrm{~m}$ interval. To get the shear waves, we change the direction of hitting parallel to the soil surface (Kaneko et al., 1990). The field data, which is in time-domain, must be converted to frequency domain (f-k spectrum) to construct dispersion curves. In 
f-k spectrum we could separate the ground roll from the signal with dip filtering (Yilmaz, 1987). Figure 6 shows only groundroll after dip filtering in time-offset curve (left panel) and in f-k spectrum (right panel). From f-k spectrum we can construct the dispersion curve (Figure 7). We can see that the dominant frequency of groundroll is about $30 \mathrm{~Hz}$ and cannot expect the existence of it in frequency range below $20 \mathrm{~Hz}$.

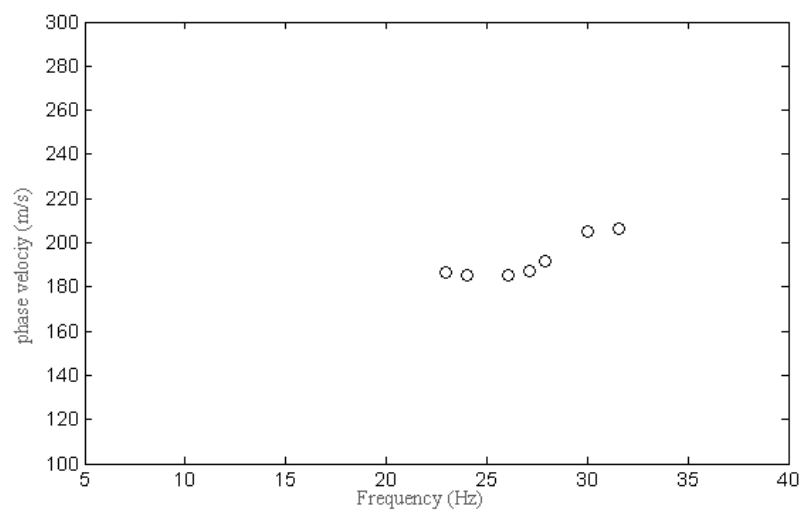

Figure 7 The dispersion curves from field experiment.

From the result of our field experiment, it was impossible to invert the dispersion curve, but at least we can conduct a numerical simulation from the field experiment data.

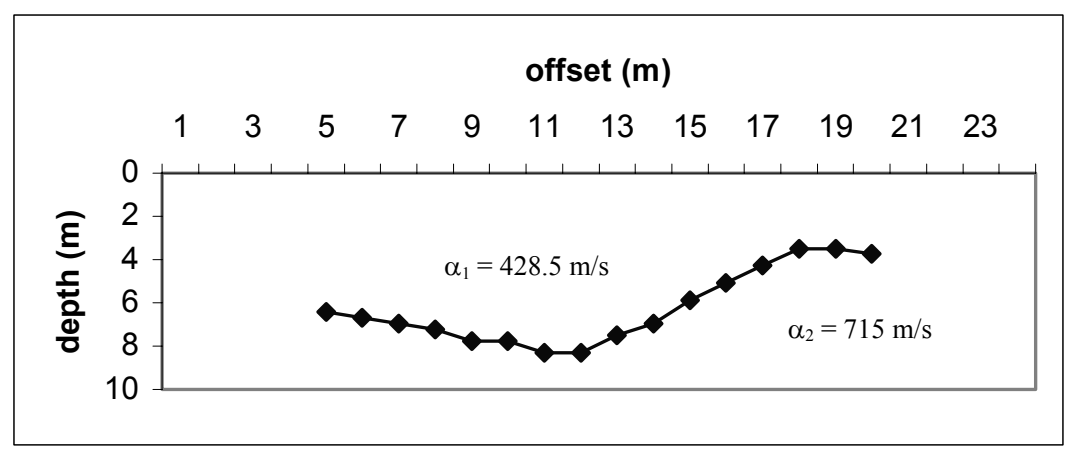

Figure 8 Depth and Velocity Profile from Seismic Refraction.

Seismic refraction result gave us the compressional waves velocities of each layer that are $\alpha_{1}=428.5 \mathrm{~m} / \mathrm{s}$ and $\alpha_{2}=715 \mathrm{~m} / \mathrm{s}$ and the thickness of top layer was about $7 \mathrm{~m}$ (Figure 8). We also could have compressional wave velocities 
from downhole method by calculating the slope of the time-distance curve (Figure 9) that are $\alpha_{1}=461.09 \mathrm{~m} / \mathrm{s}$ and $\alpha_{2}=663.7 \mathrm{~m} / \mathrm{s}$.

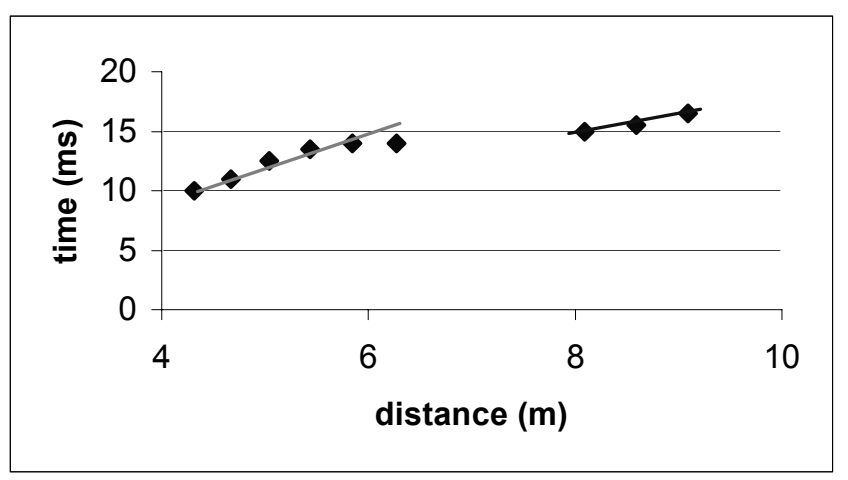

Figure 9 Time-distance curve from seismic downhole.

Setting the input from this result, we got the dispersion curve shown in figure 10. Comparing to the dispersion curve from field experiment, we found that the second layer actually could be detected in frequency range about $10 \mathrm{~Hz}$. In frequency range above $25 \mathrm{~Hz}$, we had the groundroll velocity on the top layer only. The energy source was probably too weak to penetrate deeper and the natural frequency of the geophones might be $30 \mathrm{~Hz}$.

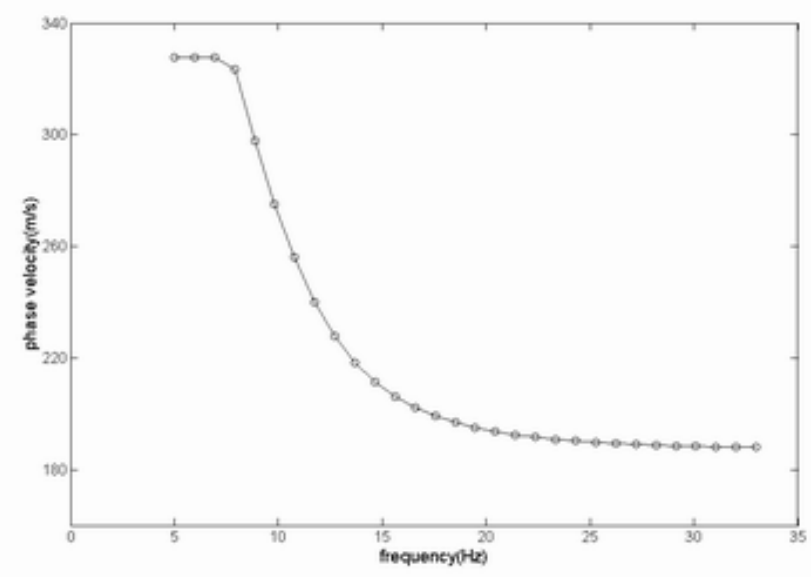

Figure 10 Numerical simulation of dispersion curve with thickness of top layer $7 \mathrm{~m}$. 


\section{$7 \quad$ Conclusions and Discussions}

By constructing dispersion curves and inverting them, we have shown that Rayleigh wave or ground roll can be used to estimate the shear wave velocity profiles. Using a seismic refraction method, we have acquired the Rayleigh wave data in time domain. The field data should be converted to frequency domain to construct dispersion curves.

For solving the instability of inversion process, we added a priori information to limit the unknown parameters. A smoothing parameter is also used to guarantee the convergence of inversion procedure and to increase the speed of convergence. Besides the numerical stability, the inversion procedure also includes the statistical stability by incorporating the uncertainty of the observational data.

We may include the thickness parameter in our inversion procedure, although it could affect in instability. We recommend to use different kind of energy sources, such as weight drop or dynamite, to acquire data from deeper layer, using common shot gather method. The geophones, also, should have lower natural frequency than what we used.

After all, we need a further research to develop this method. It is a good idea that we can have both compressional and shear wave velocities from seismic refraction method.

\section{References}

1. Aki, K. and Richards, P.G. 1980. Quantitative Seismology: Theory and Methods. W.H. Freeman and Company.

2. Chapra, S. C. and Canale, R. P. 1998. Numerical Methods for Engineers. Mc-Graw Hill.

3. Ewing, W. M., Jardetzky, S. and Press, F. 1957. Elastic Waves in Layered Media. McGraw-Hill Book Company.

4. Foti, S. 2000. Multistation Methods for Geotechnical Characterization Using Surface Waves. PhD. Diss., Politecnico di Torino.

5. Jones, R. 1962. Surface Wave Technique for Measuring The Elastic Properties and Thickness of Roads: Theoretical Development. Brit. J. Applied Geophysics vol. 13.

6. Kaneko, F., Kanemori, T., and Tonouchi, K. 1990. Low-frequency Shear Wave Logging in Unconsolidated Formations for Geotechnical Applications, in Paillet et al, Ed. 1990. Geophysical Applications for Geotechnical Investigations. ASTM. 
7. Meju, M. A. 1994. Geophysical Data Analysis: Understanding Inverse Problem Theory and Practice. Course Notes Series, Vol. 6 Society of Exploration Geophysicist.

8. Mokhtar, T. A., Hermann, R. B. and Russell, D. R. 1988. Seismic Velocity and $Q$ Model for The Shallow Structure of The Arabian Shield from Short-period Rayleigh Waves. Geophysics vol 3, no. 11.

9. Nazarian, S. and Stokoe, K.H. 1983. Nondestructive Testing of Pavement Using Surface Waves. Transportation Research Record 993.

10. Park, C. B., Miller, R.D., and Xia, J. 1999. Multichannel Analysis of Surface Waves. Geophysics Vol. 64. No.3.

11. Rikitake, S., Sato, R., and Hagiwara, Y. 1987. Applied Mathematics for Earth Scientist. Terra Scientific Publishing Company.

12. Sheriff, R. E., and Geldart, L. P. 1995. Exploration Seimology. Cambridge University Press.

13. Swelzis and Behle. 1986. Shear Wave Velocity from Rayleigh Waves, in Danbom, et al. ed. 1986. Shear Wave Exploration. Society of Exploration Geophysics.

14. Xia, J., Miller, R.D., and Park, C.B.,1999. Estimation of Near-Surface Shear-wave Velocity by Inversion of Rayleigh Waves. Geophysics vol. 64 No. 3.

15. Yilmaz, Ozdogan. 1987. Seismic Data Processing. Society of Exploration Geophysics. 